\title{
A Novel Technique and Soldering Method to Improve Performance of Transparent Polymer Antennas
}

\author{
T. Peter, Member, IEEE, R. Nilavalan, Senior Member, IEEE, Hattan F. AbuTarboush, Member, IEEE, and \\ S. W. Cheung, Senior Member, IEEE
}

\begin{abstract}
A novel technique and a nonthermal soldering method to improve the performance of AgHT-8 transparent polymer antennas are proposed in this letter. The proposed technique involves the removal of the coating layer at areas on the coplanar waveguide (CPW) ground and feed line where the connectors of the coaxial feed or legs of the SMA connectors will be attached and applying a coat of silver paint on the exposed areas before cold-soldering the coaxial connections or SMA connector legs. The nonthermal or cold soldering using electrically conductive paste enables direct soldering of the coaxial feed points or connector legs, which cannot otherwise be done with hot or thermal soldering. This type of connection greatly enhances the performance of the AgHT-8 polymer antennas compared to coaxial feed point connections through hot-soldered copper pads glued to the surface of the polymer coating. The proposed technique also gives a stronger connection bond than directly cold-soldering the feed points or connectors to the smooth surface of the AgHT-8 material. Furthermore, the copper pad connection technique also introduces additional losses contributed by the adhesive properties of the glue used. This proposed novel technique and soldering method may be extended to enhance antenna performance made from other similar transparent conductive polymers like ITO.
\end{abstract}

Index Terms-Cold soldering, copper pads, polymer, transparent, ultrawideband (UWB).

\section{INTRODUCTION}

A CHIEVING a strong connection of the coaxial connectors to provide a good electromagnetic feed to flexible polymer-based antennas is an art itself. Currently, connection of the feed points to polymer-based antennas is by thermal soldering of copper pads to the coaxial feeds or legs of the SMA connectors and gluing them to the surface of the polymer [1]. This approach has been used to avoid excessive thermal excursion, which could otherwise damage the polymer surface of the antenna, resulting in a poor connection or rendering the antenna totally useless. However, when applied to the surface of the AgHT- 8 polymer's conductive coating, this copper pad technique results in additional reflection losses that may be further

Manuscript received July 30, 2010; revised August 28, 2010; accepted August 31, 2010. Date of publication September 16, 2010; date of current version September 30, 2010.

T. Peter, R. Nilavalan, and Hattan F. AbuTarboush are with the Wireless Networks and Communications Centre (WNCC), School of Engineering and Design, Brunel University, West London UB8 3PH, U.K. (e-mail: Thomas.Peter@brunel.ac.uk).

S. W. Cheung is with the Department of Electrical and Electronic Engineering, University of Hong Kong, Hong Kong.

Color versions of one or more of the figures in this letter are available online at http://ieeexplore.ieee.org.

Digital Object Identifier 10.1109/LAWP.2010.2077271 aggravated if the adhesive used to glue the pads is too thick of a layer or provides some electrical resistance itself.

Cold soldering or nonthermal soldering using electrically conductive paste is a much better choice compared to thermal soldering as it allows direct soldering of the feed points to the antenna surface without damaging it. Although a better alternative, directly cold-soldering the coaxial feeds may also result in poor performance. This is because of the fact that the AgHT- 8 coating is a three-layered coating made up of a layer of silver sandwiched between two layers of tin oxide. Direct cold soldering will only bring the coaxial feeds in contact with the topmost layer of the coating, the tin oxide layer, which has a lower electrical conductivity compared to that of the silver layer. As such, the tin oxide surface would become the main surface along which the electromagnetic waves propagate, resulting in a poorer performance than if the feeds were connected to the sliver layer [2]. Additionally, the smooth surface of the AgHT-8 material may also result in a dry joint making the bond weak. The apparent connectivity issues in these two techniques prevent maximum power transfer of the electromagnetic energy from the coaxial feeds besides the usual power transfer loss through poor impedance matching, resulting in an overall poor antenna performance.

This letter introduces a novel technique and a nonthermal soldering approach to overcome these problems by improving the conductance of the antenna through a better conducting surface at the connectors while at the same time providing a stronger bond. This results in a better antenna performance as better power transfer of the electromagnetic energy is achieved. An ultrawideband (UWB) antenna made with transparent AgHT-8 polymer is used to demonstrate the advantages of this technique and cold soldering approach. Comparative antennas mounted by: 1) direct cold soldering; 2) copper pads using glue; and 3) copper pads cold-soldered to the antenna surface are used to further validate the effectiveness of the proposed technique and soldering method.

\section{ANTENNA DESIGN}

The UWB antenna is designed and fabricated using AgHT-8 for both the monopole and the $50-\Omega$ coplanar waveguide (CPW) feed and ground planes. The AgHT- 8 film is $0.175 \mathrm{~mm}$ thick and has a surface resistance of $8 \Omega$-Sq, which is equivalent to a conductivity $(\sigma)$ of $1.25 \times 10^{5} \mathrm{~S} / \mathrm{m}$ [1]. The AgHT- 8 film coating is made up of a transparent silver layer sandwiched between two layers of tin oxide. The antenna is mounted on a 0.175 -mm-thick polyethylene terephthalate (PET) substrate with a relative permittivity of 3.24. The antenna is designed and optimized as a semicircular disc (mushroom-shaped) for size reduction and 
TABLE I

Dimensions of the ANTENNA STRUCTURE IN Fig. 1(a)

\begin{tabular}{|c|c|c|c|c|c|c|}
\hline Parameters & L & W & Y & $\mathbf{L}_{\mathbf{1}}$ & $\mathbf{L}_{\mathbf{2}}$ & $\mathbf{W}_{\mathbf{2}}$ \\
\hline Size (mm) & 50 & 60 & 48 & 20 & 11 & 28.4 \\
\hline
\end{tabular}

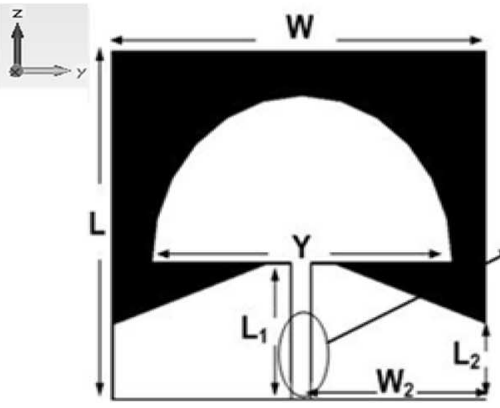

(a)
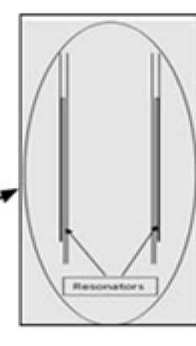

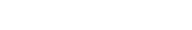

.

\begin{abstract}
Fig. 1. (a) AgHT-8 UWB antenna with resonators for impedance matching. (b) The
\end{abstract}
fed by a $50-\Omega$ CPW line as illustrated in Fig. 1(a). The radiating semicircular monopole has the radius of $24 \mathrm{~mm}$. The current in simulation is found to be mainly distributed along the edge of the semicircle monopole showing that, as already established in [3], the first resonant frequency is associated to the radiating semicircular monopole with the ultrawideband characteristic being caused by the overlapping of multiple resonant harmonics.

The CPW feed line has a width of $3 \mathrm{~mm}$ and a spacing gap of $0.1 \mathrm{~mm}$. The total dimensions of the antenna including the PET substrate are $50 \times 60 \times 0.350 \mathrm{~mm}^{3}$. The AgHT- 8 UWB antenna as such is compact and optically transparent as shown in Fig. 1(b).

The CPW grounds each have a width of $28.4 \mathrm{~mm}$, giving a total width (W) of $60 \mathrm{~mm}$ from edge to edge including the CPW feed line and the spacing gap. The gap between the CPW grounds and the bottom edge of the radiating semicircular monopole is $0.03 \mathrm{~mm}$. The ground planes are beveled [4] and have slots cut out on both walls of the ground planes along the strip gap to perform as resonators [5] to improve impedance matching and tune the lower and upper limits of the bandwidth.

The coaxial cable is connected to the feed points by soldering an SMA connector using an electrically conductive epoxy, but after removing the uppermost coating layer of the AgHT-8 film at areas on the CPW grounds and feed line where the connector legs of the SMA connector would sit, and applying a coat of silver paint on the exposed areas before actually soldering the SMA connector legs as shown in Fig. 2. It is important to test for conductivity between the silver-coated areas and the feed line and CPW grounds before actually soldering the connector. This can be done by using a simple multimeter to check for electrical continuity.

Three comparative antennas were similarly designed, but were mounted via: 1) glued copper pads at the feed points as in [1]; 2) cold-soldered copper pads; and 3) direct soldering without a silver base at the SMA connector legs.

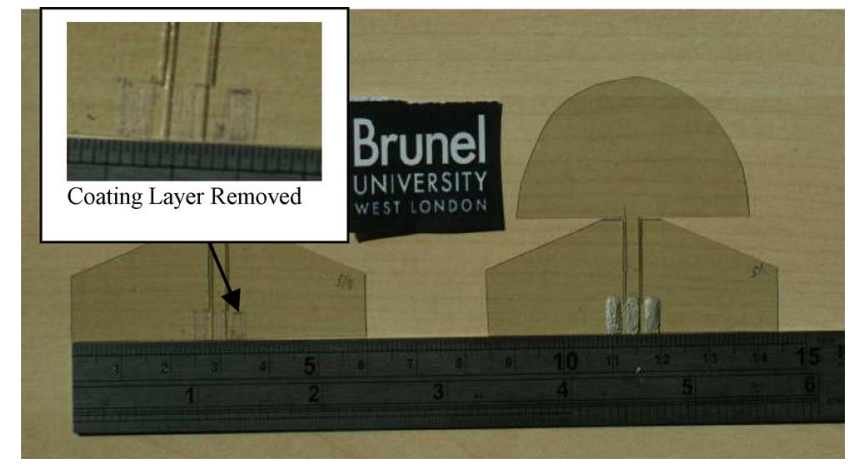

Fig. 2. (Left) Antenna with the coating removed. (Right) Antenna after application of the conductive silver paint.

\section{Measurement And Simulation Results}

The antennas were designed and simulated on CST Microwave Studio. The performance of the proposed approach was verified by fabricating the monopole UWB CPW-fed antenna and measuring the return loss. The measured return losses of the four fabricated antennas are comparatively shown with the simulated result of the designed antenna with a $50-\Omega$ port in Fig. 3. The return loss pattern of the silver-based antenna is the most similar to that of the simulated return loss, demonstrating that the proposed approach enables us to achieve a result closest to the ideal or designed performance.

The silver-based antenna also showed comparatively better return loss performance than the one without. The return loss for the antenna fed via cold-soldered copper pads showed better performance than the one that had glued copper pads. This was probably due to the adhesive that was used to glue the copper pads to the antenna; it could have contributed some electrical resistance. It was also observed that compactness of the antenna gave it rigidity for independent mounting without any additional support. Of the four antennas, the antenna with the silver base 


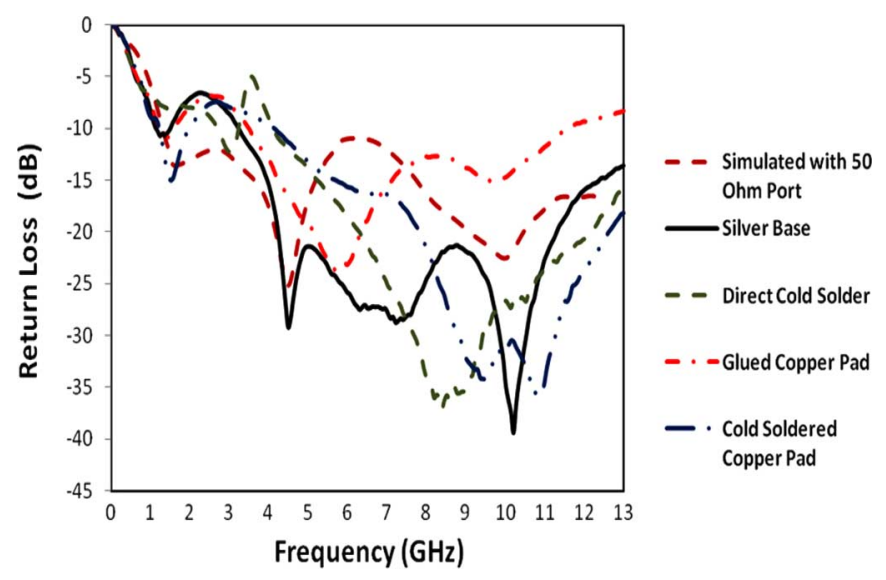

Fig. 3. Measured return loss of the antennas versus simulated return loss with a $50-\Omega$ port.

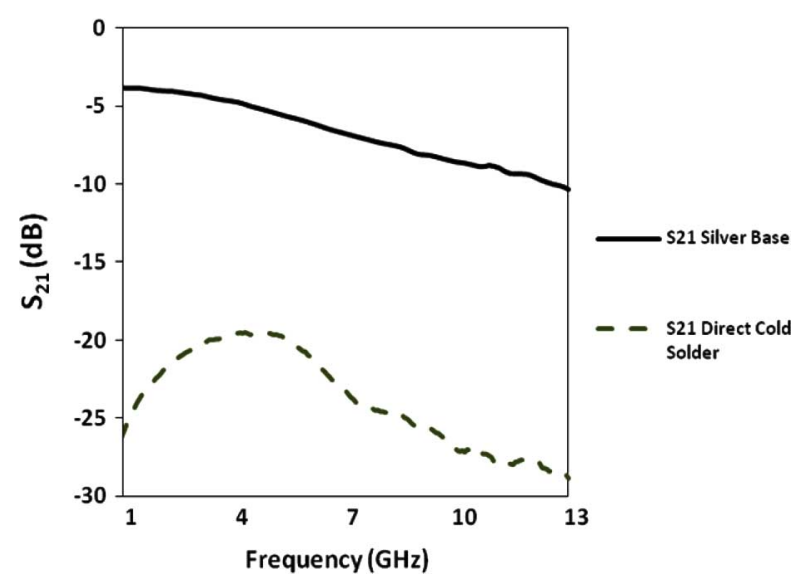

Fig. 4. Measured $\mathrm{S}_{21}$ gain of the silver-base technique compared to the direct cold-solder technique for a CPW AgHT-8 strip of $20 \mathrm{~mm}$ length.

was found to have a stronger bond by virtue of the entrenched sublayer foundation of the silver base. This made the silverbased antenna a better candidate of the four antennas while at the same time validating the effectiveness of the novel technique proposed in this letter.

An experimental analysis carried out to evaluate the proposed connection technique by connecting the SMA connector to a CPW line of $20 \mathrm{~mm}$ and back to an SMA connector, and measuring the $\mathrm{S}_{21}$, showed that the proposed technique further improved the performance of the connection. In this experiment, only the proposed silver-base technique and the direct coldsolder approach were compared. The results of the experiment are shown in Fig. 4. Although this experimental $\mathrm{S}_{21}$ analysis quite substantiates the claim of the proposed technique, the aim of the letter was to further analyze the use of the proposed technique on the performance of the AgHT- 8 transparent polymer antenna.

Efficiency, gain, and radiation pattern measurements of the four fabricated prototypes tested on Satimo's antenna measurement equipment, StarLab, further affirmed the findings based on the return loss. The measured efficiencies of the four prototype antennas are shown in Fig. 5, while the peak gains of the antennas are shown in Fig. 6. The radiation patterns at 3

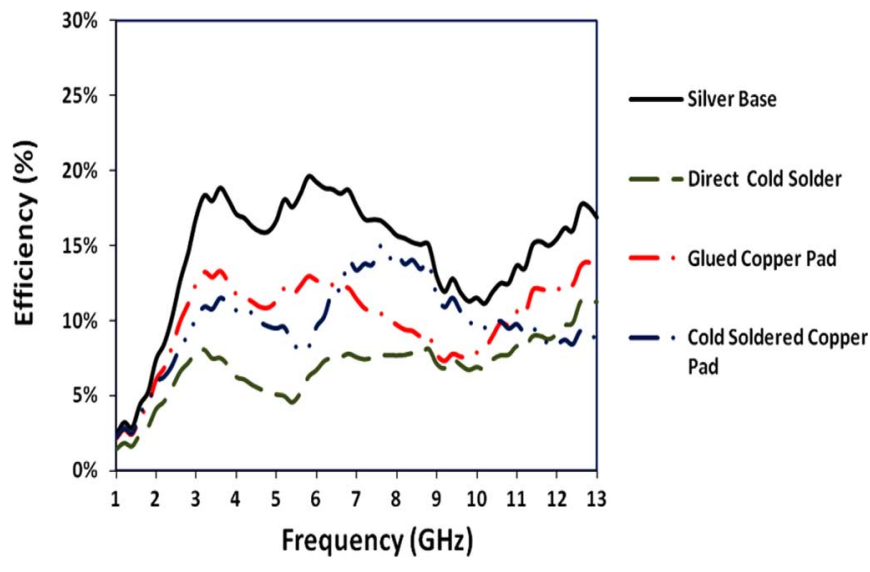

Fig. 5. Measured efficiency of the four antennas.

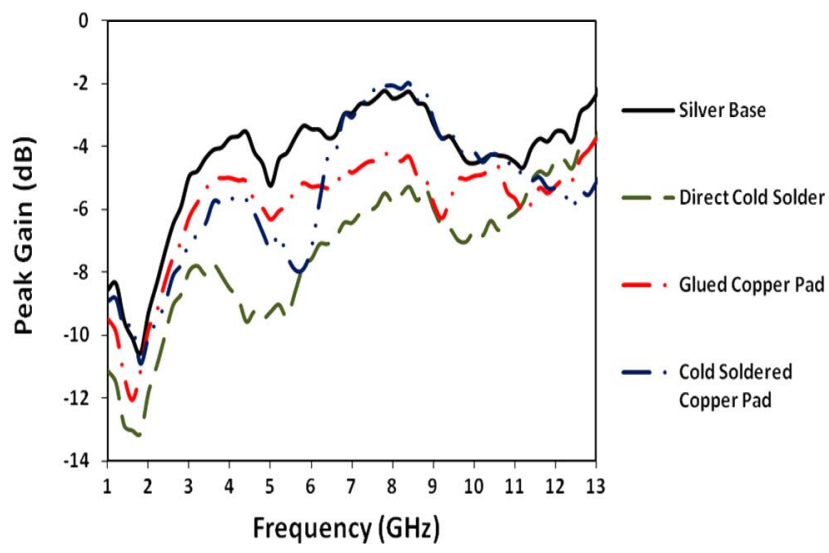

Fig. 6. Measured peak gain of the four antennas.

and $7 \mathrm{GHz}$ in the vertical $y z$ plane and horizontal $x y$ plane are shown in Fig. 7. The silver-based antenna demonstrates a smaller cross-polar pattern in the vertical plane compared to the other antennas besides also demonstrating an omnidirectional characteristic in the horizontal plane.

\section{CONCLUSION}

A novel technique and a nonthermal soldering method were proposed to improve the performance of polymer-based antennas made of transparent conductive materials by applying a silver base on the exposed subsurface at the coaxial connections before cold-soldering them with conductive epoxy. The effectiveness of the proposed technique was shown through $\mathrm{S}$-parameter simulation and measurements of a monopole AgHT-8 UWB antenna with the feed connected using conductive epoxy with a silver base and comparative antennas without a silver base or using copper pads that were glued or cold-soldered only.

Additional experimental validation of the connection performance of the proposed silver-base technique using the standard $\mathrm{S}_{21}$ method for connector performance indication was also carried out. The results showed that the proposed technique greatly improved the connection performance. The measured return loss, efficiency, and peak gain for the silver-based antenna show better results over that without the silver base and that of the copper pad antennas as demonstrated in Figs. 3, 5, and 6, 

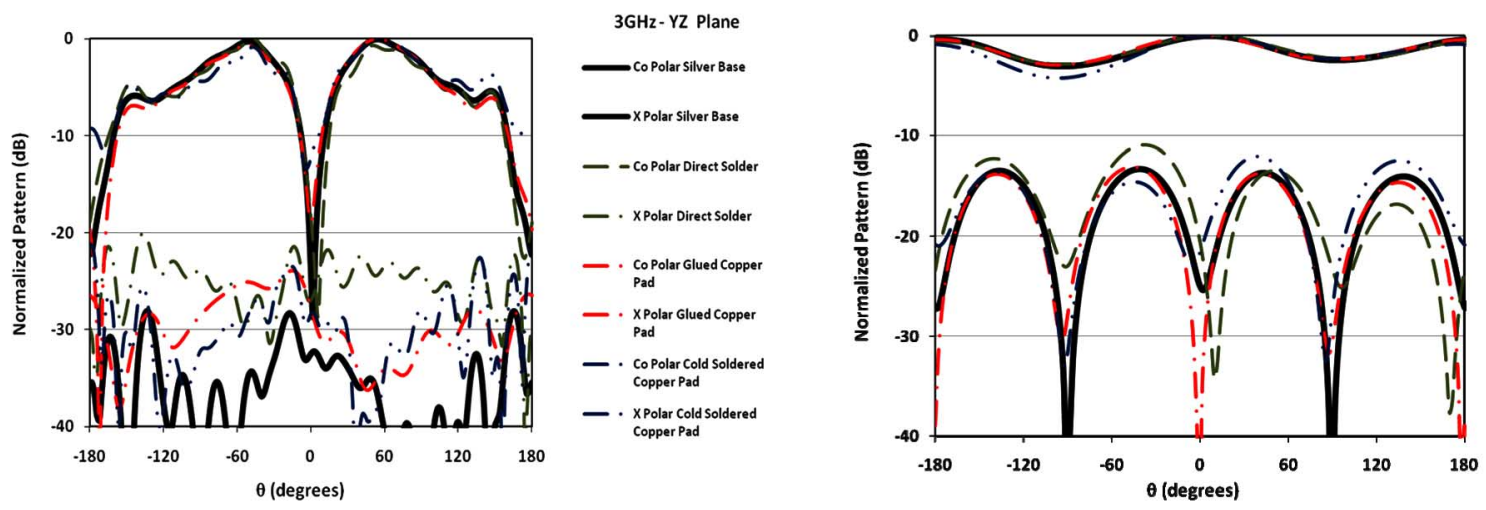

$3 \mathrm{GHz}-\mathrm{XY}$ Plane

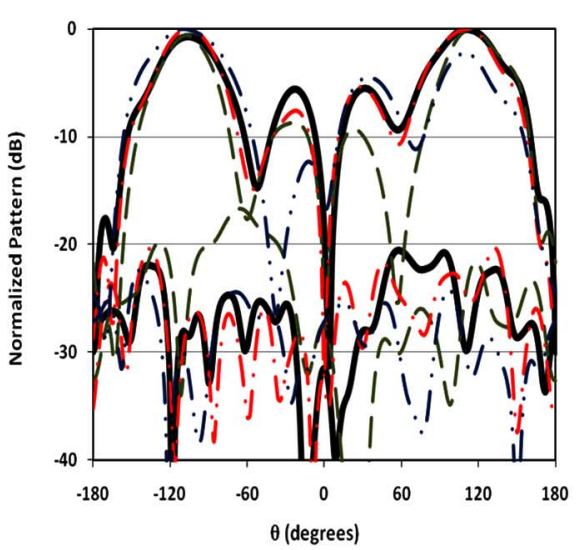

7GHz-YZ Plane
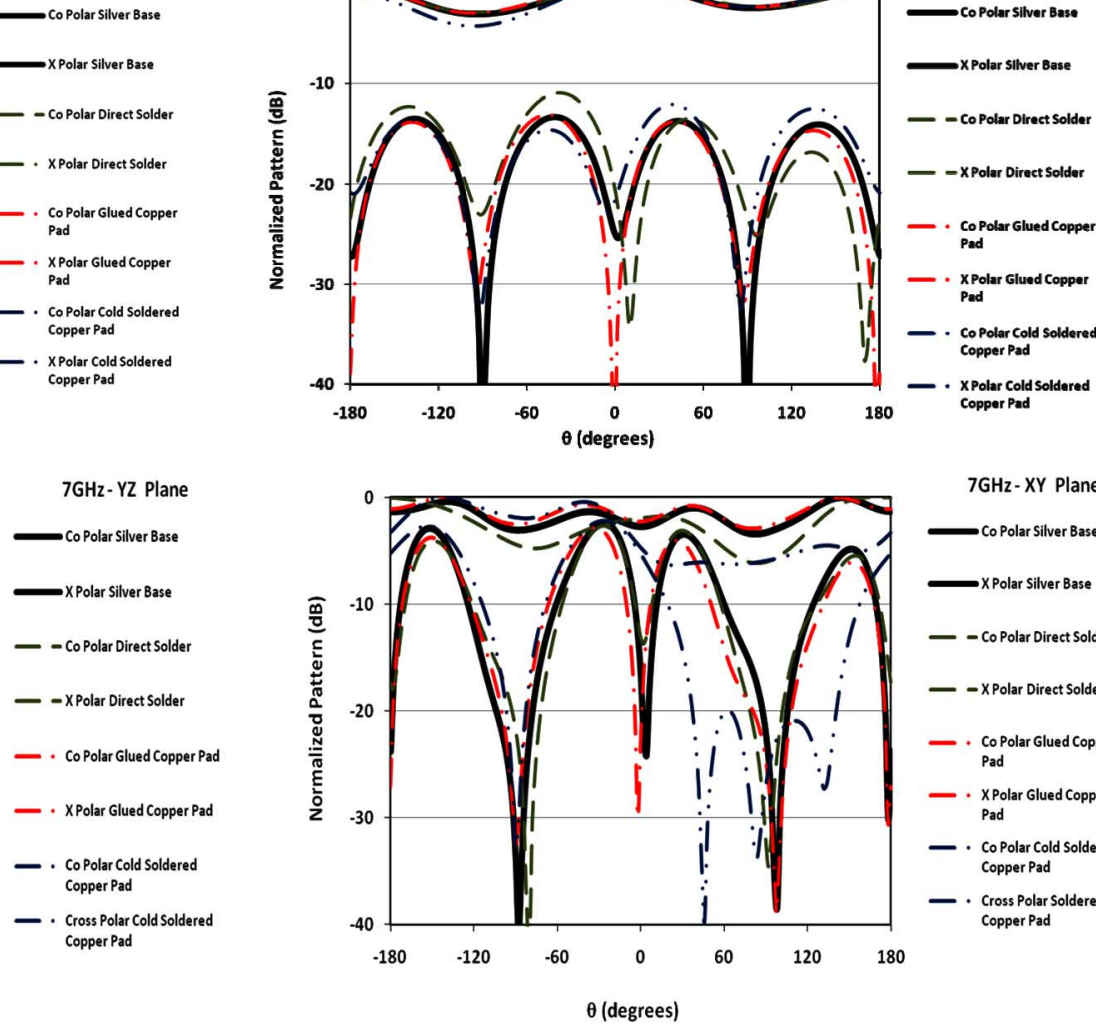

7GHz-XY Plane
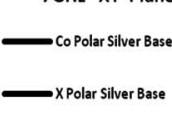

- Co Polar Direct Solder

— X Polar Direct Solder - Co Polar Glued Copper
Pad

- x Xolar Glued Copper Pad

- co Polar Cold Soldered Copper Pad

- Cross Polar Soldered Copper Pad

Fig. 7. Measured radiation patterns in the $y z$ and $x y$ planes for the four antennas.

thus validating the effectiveness of both the technique and the nonthermal soldering approach.

The proposed technique and soldering method can be employed in the design and fabrication of antennas using similar transparent conductive polymer film materials.

\section{ACKNOWLEDGMENT}

The authors wish to acknowledge CPFilms, Portsmouth, U.K., for its support in providing the AgHT-8 films used in this research.

\section{REFERENCES}

[1] J. S. Hyok, Y. H. Tsung, D. F. Sievenpiper, P. H. Hui, J. Schaffner, and E. Yasan, "A method for improving the efficiency of transparent film antennas," IEEE Antennas Wireless Propag. Lett., vol. 7, pp. 753-756, 2008.

[2] CST 2010-Properties Material Library.

[3] C. Yu, W. Hong, and Z. Kuai, "Multiple stopbands ultra wide band antenna," in Proc. Int. Conf. Microw. Millim. Wave Technol., Apr. 2008, vol. 4, pp. $1872-1874$.

[4] M. J. Ammann, "Control of the impedance bandwidth of wideband planar monopole antennas using a beveling technique," Microw. Opt. Technol. Lett., vol. 30, no. 4, pp. 229-232, 2001.

[5] T. Peter and R. Nilavalan, "Study on the performance deterioration of flexible UWB antennas," in Proc. LAPC, 2009, pp. 669-672. 